\title{
Le «complexe»
}

\section{Sergentomyia (Sergentomyia) antennata}

(Newstead, 1912)

\author{
Remarques systématiques et écologiques \\ à propos de 948 exemplaires récoltés dans le Sud-marocain
}

\author{
par N. LEGER *, J.-A. RIOUX **, H. CROSET **, M. CADI SOUSSI *** \\ et N. BENMANSOUR ***; \\ (collaboration technique: M. MAISTRE et Y. JoNQUET) \\ * Laboratoire de Parasitologie, Faculté de Pharmacie, \\ 4, avenue de l'Observatoire, F 75006 Paris \\ ** Laboratoire d'Ecologie médicale et de Pathologie parasitaire ( $\mathrm{P}^{\mathrm{r}} \mathrm{J}$.-A. RIoux) \\ Faculté de Médecine, rue Auguste-Broussonnet, F 34000 Montpellier \\ *** Faculté de Médecine, Rabat, Maroc
}

\section{Résumé.}

La capture, au sud des Atlas, d'un lot important de Sergentomyia cf. antennata (Newstead, 1912) permet aux auteurs de discuter, sinon de réfuter, les critères biométriques utilisés pour individualiser les «formes» signatipennis, cincta, occidentalis et antennata s. st. La distribution des dents cibariales couvre en effet les valeurs données par les inventeurs pour définir ces formes. En attendant la mise au point de nouveaux critères taxonomiques, le binôme $S$. antennata s.l. est proposé pour désigner l'ensemble des populations d'Afrique du Nord.

\section{Summary.}

The complex Sergentomyia (Sergentomyia) antennata (Nevstead, 1912) systematic and ecological notes on 948 specimens found in south Morocco.

The capture of considerable Sergentomyia cf. antennata (Newstead, 1912) south of the Atlas gave the authors to discuss and even to refute the usual biometrical criteria for the subspecies: signatipennis, cincta, occidentalis and antennata s. st. Indeed the distribution of cibarial teeth in this population covers the numbers given by the original authors for defining these different forms. Until new taxonomic criteria are found, the binominal name $S$. antennata s. 1 . is proposed for the whole of the population of North Africa. 
Le groupe «antennata » dont le chef de file, Sergentomyia antennata a été décrit du Ghana par R. Newstead (1912), se présente actuellement comme un « complexe systématique » assez confus qui réunit une foule de formes affines, considérées suivant les auteurs tantôt comme de bonnes espèces, tantôt comme des sous-espèces, voire des populations plus ou moins ségrégées. Aussi bien, nous paraît-il intéressant d'apporter une nouvelle contribution à l'étude de ce problème, en discutant les résultats d'une analyse biométrique réalisée sur un lot de 704 exemplaires $q$ capurés en 1972 au cours d'une mission effectuée dans le sud du Maroc (1).

La présente note comporte trois chapitres. Dans le premier nous retraçons l'historique du groupe «antennata ». Nous consacrons le deuxième à l'interprétation des données biométriques fournies par l'examen de nos exemplaires. Le dernier précise les caractéristiques écologiques des stations marocaines inventoriées à ce jour.

\section{Rappel historique.}

Deux étapes bien tranchées jalonnent la connaissance systématique des Phlébotomes. Dans la première, antérieure à 1928, les « inventeurs » utilisent exclusivement la morphologie externe des imagos et partant, commettent de multiples erreurs. De cette époque datent de nombreux synonymes.

Au cours de l'étape suivante, les auteurs s'efforcent de remettre un certain ordre en s'appuyant sur l'observation des structures internes (spermathèques, armatures pharyngienne et cibariale). Plusieurs binômes disparaissent, d'autres sont restaurés. La taxonomie s'enrichit de genres et de sous-genres nouveaux.

L'individualisation du sous-genre Sergentomyia Parrot et França en particulier procède de cette nouvelle orientation; appartenant précisément à ce sous-genre, le groupe antennata est analysé à son tour (2).

Son histoire débute en 1912 où, à partir d'un exemplaire femelle capturé à Salaga (Ghana), R. Newstead décrit, sous le binôme Phlebotomus antennatus, une espèce caractérisée par la brièveté des pattes, l'aspect massif des segments antennaires pourvus d'épines géniculées, du $3^{\circ}$ au $15^{\circ}$.

En 1920, ce même auteur décrit, également du Ghana, une nouvelle espèce, $P$. signatipennis, se singularisant par une pilosité exceptionnelle. Dans le même article, il rapporte de nouvelles captures de $P$. antennatus $\sigma^{t}$ et + sans préciser toutefois les affinités systématiques des deux taxons.

En 1928, J. Sinton met l'accent sur l'intérêt systématique des spermathèques et de l'armature buccale. L'examen des spécimens-types lui permet de clarifier la classification des espèces asiatiques. A juste titre plusieurs binômes sont mis en synonymie. Mais assez malencontreusement il inclut $P$. antennatus dans les variétés de $P$. minutus.

(1) RCP 249, C.N.R.S.: *Etude de certains milieux du Maroc et de leur évolution récente. »

(2) Nous remercions MM. E. Abonnenc et D. J. Lewis qui ont mis à notre disposition leur importante collection et nous ont aidés de leurs conseils. 
Ce point de vue, adopté par divers auteurs, va compromettre l'analyse correcte du complexe antennata.

Ainsi L. Parrot (1930) mentionne P. minutus var. antennatus dans une série du Congo belge. Cette dernière contribution est cependant positive dans la mesure où pour la première fois, le nombre de dents du cibarium (ici 25) et la forme de l'aire pigmentée sont retenus comme critères d'identification.

En 1931, dans son article "On african sandflies», O. Theodor se range à son tour à l'opinion de J. Sinton. Dans la liste des 24 espèces rapportées, il signale l'existence de quatre taxons douteux, parmi lesquels $P$. minutus var. antennatus. Pour lui, $P$. signatipennis (3) est synonyme de $P$. minutus Rondani, 1843.

La confusion ne fait que s'affirmer lorsque O. Theodor (1931-1933) entreprend de redécrire (4) P. minutus s. st. (exemplaires de Palestine, de Mésopotamie et des Indes), $P$. minutus var. signatipennis (exemplaires du Soudan) et $P$. minutus var. antennatus (exemplaires du Ghana). Dans un deuxième article, il propose une variété nouvelle, $P$. minutus var. signatipennis (exemplaire du Soudan), et pense que $P$. minutus var. antennatus, décrit du Congo par L. Parrot, serait à reventiler entre les variétés signatipennis et occidentalis.

En fait, les caractères taxonomiques invoqués ne sont pas très convaincants, et, de l'avis même de l'auteur, les exemplaires étiquetés \& $P$. minutus $\gg \mathrm{s}$. st. seraient indiscernables des $P$. signatipennis. Cette constatation l'amène fort judicieusement à réclamer un nouvel examen des exemplaires-types.

L. Parrot, le premier, tente de clarifier une situation particulièrement confuse. S'appuyant sur l'examen de $P$. minutus var. signatipennis récoltés au Hoggar et en Algérie (1941 et 1942), il replace $P$. signatipennis au rang d'espèce à laquelle il rattache $P$. sanneri Galliard et Nitzulescu, 1931. Pour lui, le Phlébotome signalé par S. Adler et $\mathrm{O}$. Theodor de Palestine, sous le binôme « $P$. minutus , doit être regroupé au côté de $P$. fallax, sans toutefois être confondu avec ce dernier ( $P$. theodori nov.).

Dès lors, la distinction entre le groupe $P$. minutus et ce qui deviendra ultérieurement le groupe antennata, est nettement établie. Ce n'est plus que sur la valeur des diverses espèces ou variétés que vont porter les discussions.

A partir de 9 femelles récoltées à Djibouti, L. Parrot et A. Martin (1944) décrivent $P$. cinctus qu'ils différencient des autres espèces du groupe antennata $(P$. signatipennis, $P$. occidentalis et $P$. antennatus) par l'aspect et le nombre des dents buccales, le dessin de la plage pigmentée, la longueur relative des épines géniculées du $4^{\circ}$ article antennaire et la taille des épines de l'armature pharyngienne.

(3) D'après l'examen des exemplaires déposés au "London School of Tropical Medecine».

(4) Faute d'avoir pu examiner l'holotype italien de A. Rondani (redécrit par G. Sacca en 1947) ou les cotypes maltais de Newstead. O. Theodor s'appuie d'une part sur des arguments biogéographiques et d'autre part sur l'avis de J. Sinton affirmant la similitude des coiypes maltais et des exemplaires palestiniens. 
Dans un lot de Phlébotomes provenant du Soudan anglo-égyptien, L. Parrot (1948) retrouve $P$. cinctus, associé à $P$. occidentalis. Après avoir discuté une nouvelle fois des critères de différenciation, l'auteur ne retient plus que le nombre de dents du cibarium : 14 à $20 \mathrm{chez} P$. cinctus, 22 à $26 \mathrm{chez} P$. signatipennis, 26 à $32 \mathrm{chez} P$. occidentalis.

A

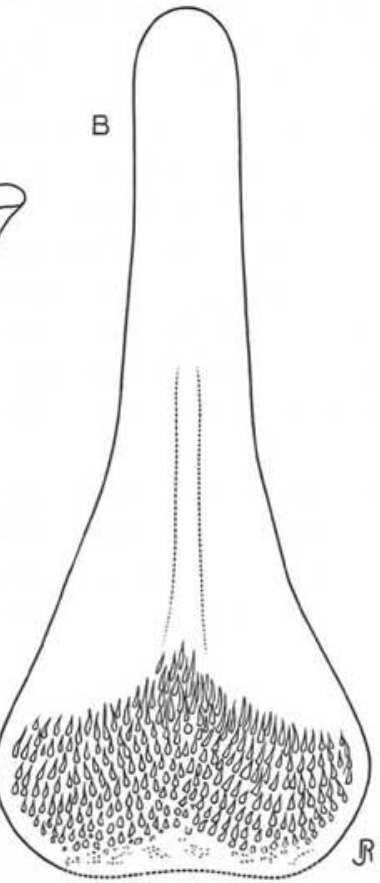

Fig. 1. - Sergentomyia antennata (Newstead, 1912). Cibarium (A) et pharynx (B).

Examinant un lot provenant du Sénégal et du Soudan, L. Parrot, P. Mornet et J. Cadenat (1945) décrivent, sous le binôme $P$. dubius, une espèce qu'ils rapprochent provisoirement de $P$. antennatus Newstead. A cette occasion les auteurs demandent de statuer sur la validité de cette espèce. Pour eux, le problème systématique ne sera réglé qu'après l'examen des types de Newstead et des exemplaires indiens étiquetés $P$. minutus antennatus par J. Sinton (1927).

Un tel examen est effectivement réalisé en 1949 par R. Kirk et D. J. Lewis : l'holotype et les syntypes $ᄋ$ de $P$. antennatus déposés au British Museum offrent un cibarium pourvu de 26 à 28 dents et un pharynx cordiforme. Il devient alors évident que $P$. signatipennis et les formes apparentées ne peuvent plus être considérés comme des variétés de $P$. minutus, mais doivent être regroupés autour de $P$. antennatus. En fait pour ces auteurs il n'existerait qu'une espèce, intégrant les cinq variétés suivantes: 
P. antennatus antennatus Newstead, 1912 ;

$P$. antennatus signatipennis Newstead, 1920 ;

P. antennatus occidentalis Theodor, 1933 ;

P. antennatus cinctus Parrot et Martin, 1944 ;

P. antennatus dubius Parrot, Mornet et Cadenat, 1945.

La publication de R. Kirk et D. J. Lewis amène L. Parrot (1951) à préciser une dernière fois sa position vis-à-vis du groupe antennatus. D'une manière générale, il se déclare favorable à cette dernière analyse. Pour lui, $P$. signatipennis, $P$. occidentalis et $P$. cinctus doivent effectivement prendre rang de variétés de $P$. antennatus. Cependant il maintient la validité de $P$. dubius en raison du dimorphisme très accusé des dents cibariales et de la forme de la plage pigmentée (dépourvue de «pointe »).

En 1972, E. Abonnenc confirme en partie les conclusions précédentes. Il regroupe cependant $P$. signatipennis et $P$. occidentalis sous le binôme $P$. antennatus et considère $P$. cinctus et $P$. dubius comme de bonnes espèces. Au sein du groupe antennatus, cet auteur distingue ainsi trois espèces :
$P$. antennatus Newstead, 1912 ;
P. cinctus Parrot et Martin, 1944 ;
P. dubius Parrot, Mornet et Cadenat, 1945.

\section{Appartenance systématique des exemplaires marocains.}

Les femelles capturées au Maroc se caractérisent par:

- l'aspect cordiforme (fig. 1, A) de l'armature pharyngienne qui permet de les rattacher au groupe fallax-antennata (5);

- le groupement des dents pharyngiennes en une plage homogène, caractère qui distingue le complexe antennata de $S$. fallax chez lequel les dents périphériques sont de taille nettement plus forte que les centrales (fig. 1, B) ;

- le monomorphisme des dents cibariales et la présence, à la partie antérieure de l'aire pigmentée, d'un prolongement en «casque à pointe» (fig. 1, A), caractères qui permettent d'éliminer $S$. dubia.

L'histogramme, construit à partir de 704 ㅇ (fig. 2), montre que le nombre de dents varie de 12 à $24(\bar{x}=18,73 \pm 0,13)$ c'est-à-dire englobe les valeurs proposées par L. Parrot pour $S$. signatipennis (22 à 26 dents) et $S$. cincta (14 à 20 dents).

Au surplus, les numérations effectuées à partir de deux autres lots, provenant respectivement de la Mauritanie et du Ghana, donnent une distribution assez voisine (fig. 2). Ainsi les exemplaires du Ghana, capturés dans une région proche de la station princeps de $S$. antennata, présentent 17 à 26 dents $(\bar{x}=20,67 \pm 0,20)$.

(5) A l'opposé de $S$. fallax, l'échancrure postéro-médiane đu pharynx a tendance à s'estomper chez les $S$. antennata montés au baume. 


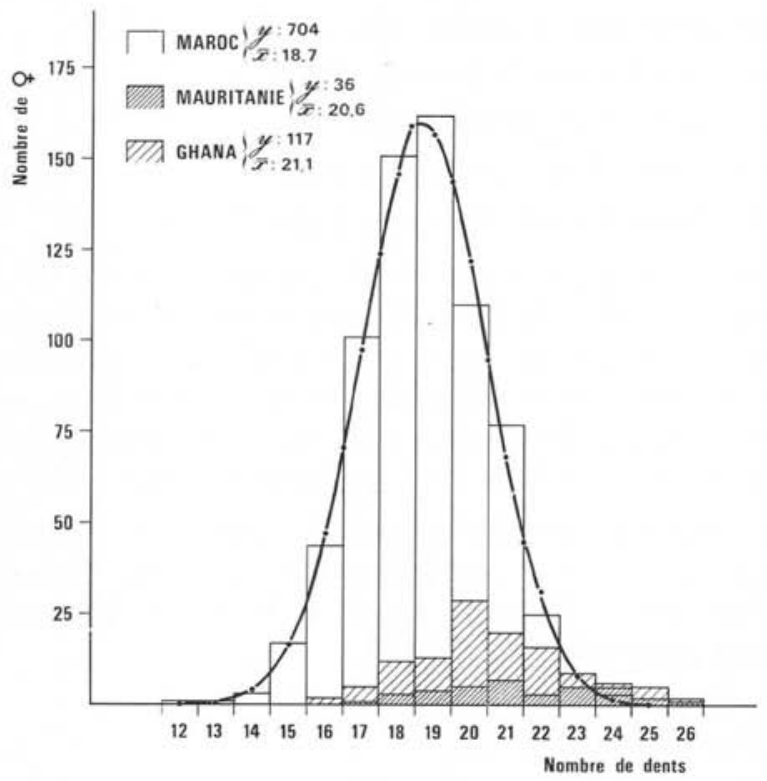

FIG. 2. - Sergentomyia antennata (Newstead, 1912). Distribution du nombre de dents du cibarium dans trois populations, marocaine, mauritanienne et ghanéenne.

Cette étude remet donc en question la réalité biosystématique des taxons antennata, cincta et signatipennis, autrement dit amène à réexaminer les limites et le contenu du «complexe» $S$. antennata. Il n'est pas impossible qu'il s'agisse d'une même espèze se comportant comme un «cline» tout au moins pour ce qui concerne la fréquence géographique des dents cibariales.

Quoi qu'il en soit, en l'absence de données nouvelles, il nous paraît plus prudent de rassembler les exemplaires récoltés au Maroc sous le binôme $S$. antennata (Newstead, 1912) s.l.

\section{Données écologiques.}

Les stations marocaines de $S$. antennata se situent dans leur ensemble au Sud de la chaîne des Atlas (fig. 3). Ainsi, au cours de la mission itinérante réalisée en août et septembre 1972, de Meknès à Ouarzazate, via Merzouga, les premières stations fertiles ont été observées aux environs de Ksar-es-Souk (tableau VI). La densité des captures (6) relativement faible dans cette zone a nettement augmenté à partir d'Erfoud, en sous-climat saharien tempéré (tableau I).

(6) Il s'agit de captures au piège adhésif selon la méthode des «itinéraires-transects » définie antérieurement (J.-A. Rioux et coll., 1967). 


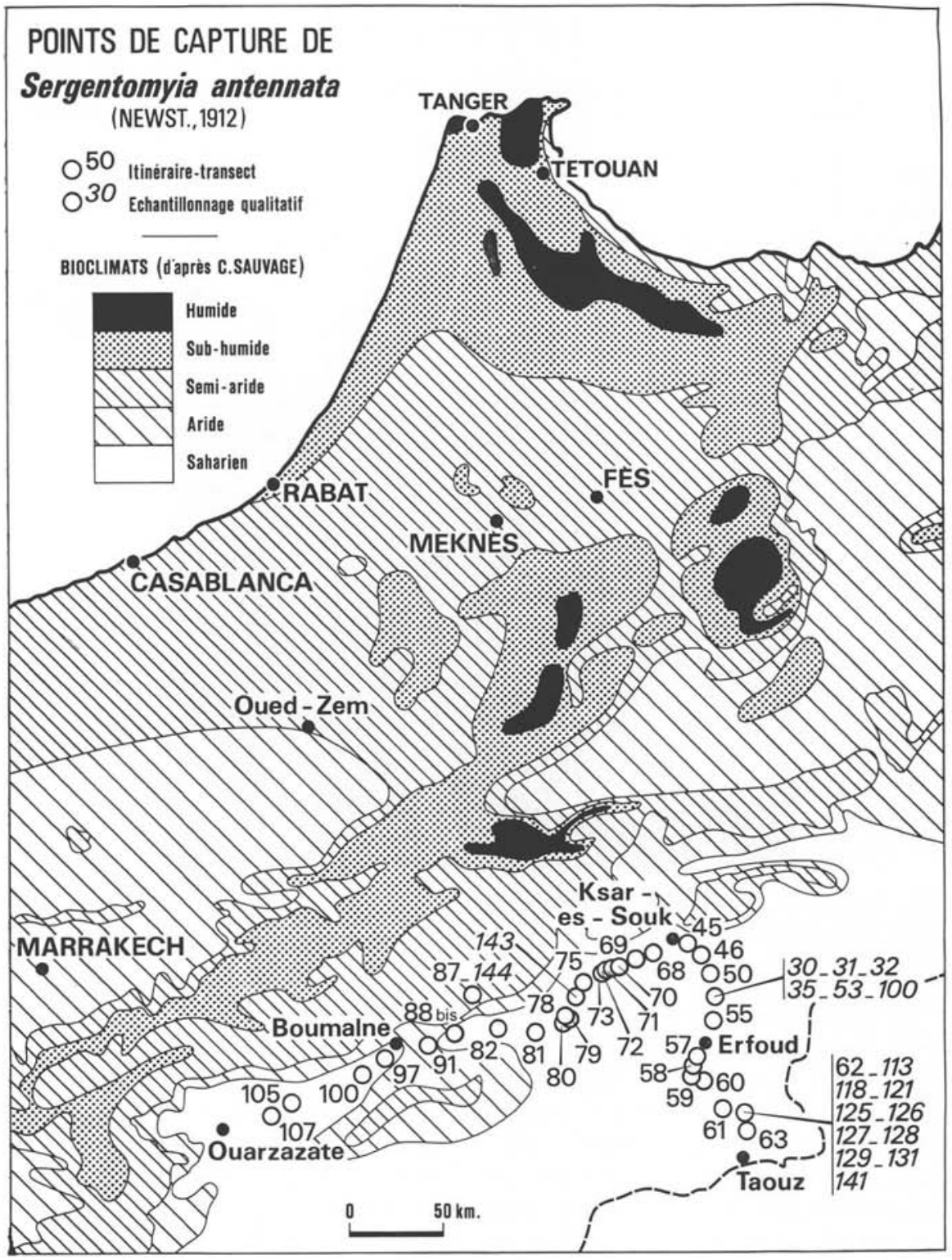

FIG. 3. 
Tableau I. - Densités de Sergentomyia antennata (Newst.) exprimées selon les étages bioclimatiques en nombre de Phlébotomes par $m^{2}$ de piège. L'espèce apparaît à l'étage aride (frais) et devient abondante à l'étago saharien (tempéré)

\begin{tabular}{|c|c|c|c|c|c|}
\hline \multirow{2}{*}{$\begin{array}{c}\text { Etages } \\
\text { bioclimatiques }\end{array}$} & \multirow{2}{*}{$\begin{array}{l}\text { Surfaces } \\
\text { de pièges }\end{array}$} & \multicolumn{3}{|c|}{ Sergentomyia antennata } & \multirow{2}{*}{$\begin{array}{l}\text { Densités } \\
\mathrm{Ph} 1 / \mathrm{m}^{2}\end{array}$} \\
\hline & & $q$ & $\delta$ & Total & \\
\hline aride frais $\ldots \ldots \ldots \ldots$ & $11,84 \mathrm{~m}^{2}$ & 1 & 4 & 5 & 0,42 \\
\hline saharien frais $\ldots \ldots \ldots \ldots$ & $83,36 \mathrm{~m}^{2}$ & 166 & 47 & 213 & 2,56 \\
\hline saharien tempéré $\ldots \ldots \ldots$ & $30,64 \mathrm{~m}^{2}$ & 131 & 125 & 256 & 8,38 \\
\hline
\end{tabular}

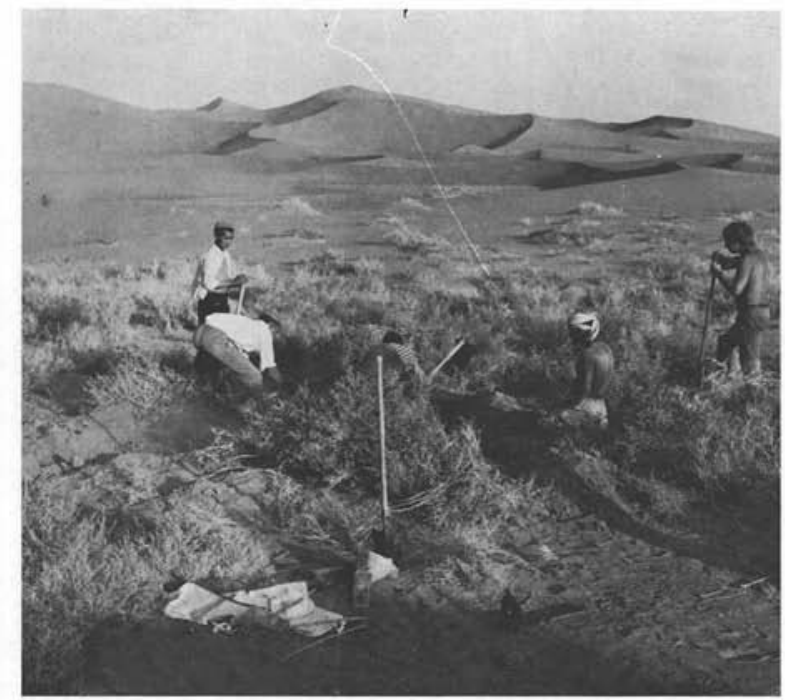

Fig. 4. - Dunes à Salsolacées. Terriers de Psammomys obesus Cretzchmar, biotope de Sergentomyia antennata (Newstead, 1912). Le creusement du terrier permet la capture du Rongeur, difficile par les méthodes traditionnelles de piégeage (Merzouga le 17-9-72).

L'analyse mésologique des stations inventoriées permet de dégager assez clairement le biotope optimal de $S$. antennata.

Ainsi que l'a souligné R. Durand-Delacre dans son étude sur les Phlébotomes de la région de Beni-Ounif-de-Figuig, ce biotope est constitué par les terriers de Gerbillidés (Meriones libycus, Psammomys obesus, Gerbillus hirtipes, Gerbillus campestris, etc...). Ces terriers sont le plus souvent creusés en terrain limoneux (Rissani, Erfoud) ou sableux (Merzouga) (fig. 4 et 5) plus rarement en terrain rocheux (Tinedjad). Les plus fortes densités phlébotomiennes s'observent dans les gîtes situés au voisinage des nappes aquifères (zones d'épandage, berges d'oued), des réseaux d'irrigation (canaux d'alimentation et de collature) ou des hautes dunes à pouvoir de rétention hydrique élevé (tableau VI). Les femelles de $S$. antennata dominent largement dans les pièges adhésifs $(99,8 \%)$ tendus à l'entrée de galeries (tableaux II, V et VI), constatation qui confirme le comportement essentiellement rodontophile de l'espèce. 


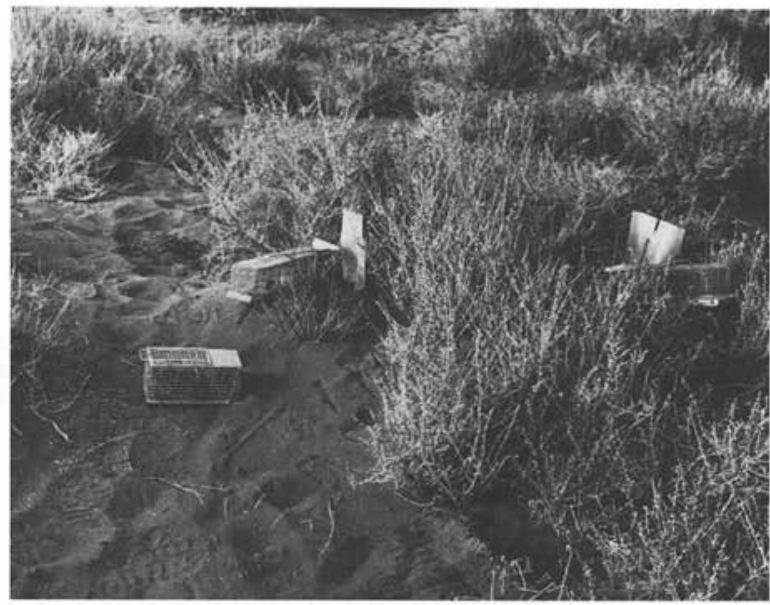

FIG. 5. - Détails de la station précédente. Deux pièges adhésifs à Phlébotomes et deux pièges métalliques à Rongeurs sont tendus devant les orifices des terriers. Au premier plan, un Psammomys obesus capturé par creusement.

TABlEAU II. - Espèces associées à Sergentomyia antennata (Newst.) dans les terriers de Rongeurs. Noter la relative abondance de Phlebotomus papatasi (Rond.). Par contre, les représentants des Paraphlebotomus sont rares (Phlebotomus alexandri Sinton, 1928) ou très rares (Phlebotomus sergenti Parrot, 1917)

\begin{tabular}{|c|c|c|c|c|}
\hline Phlébotomes & $q$ & 8 & Total & $\%$ \\
\hline Sergentomyia antennata ..... & 513 & 52 & 565 & 79,19 \\
\hline Phlebotomus papatasi ...... & 110 & 60 & 170 & 21,12 \\
\hline Sergentomyia fallax ........ & 25 & 15 & 40 & 4,97 \\
\hline Phlebotomus alexandri ..... & 6 & 6 & 12 & 1,49 \\
\hline Phlebotomus bergeroti ..... & 2 & 2 & 4 & 0,50 \\
\hline Sergentomyia christophersi .. & . & 4 & 4 & 0,50 \\
\hline Sergentomyia clydei ........ & 2 & 1 & 3 & 0,37 \\
\hline Sergentomyia dreyfussi ..... & . & 2 & 2 & 0,25 \\
\hline Sergeniomyia minuta s. sp. .. & . & 2 & 2 & 0,25 \\
\hline Phlebotomus longicuspis .... & . & 2 & 2 & 0,25 \\
\hline Phlebotomus sergenti ...... & - & 1 & 1 & 0,12 \\
\hline
\end{tabular}

S. antennata est exceptionnellement observé dans les maisons habitées. Il est toutefois attiré par la lumière ainsi qu'en témoignent les récoltes réalisées à AoufousBlaghma, les 8 et 9 juillet 1972 (tableau III). 
TABleAU III. - Sergentomyia antennata (Newst.). Résultats des captures au piège lumineux réalisées à proximité du bivouac de Aoufous-Blaghma le 8 et 9 septembre 1972

\begin{tabular}{|c|c|c|c|c|c|}
\hline \multirow{2}{*}{ Dates } & \multirow{2}{*}{ Biotopes } & \multirow{2}{*}{ Modes de capture } & \multicolumn{3}{|c|}{ Sergentomyia antennata } \\
\hline & & & q & $\sigma$ & Total \\
\hline 8 septembre 1972 & $\begin{array}{l}\text { Camion-laboratoire } \\
\text { installé sur le « reg » }\end{array}$ & $\begin{array}{l}\text { Capturè manuelle à } \\
\text { l'intérieur du véhi- } \\
\text { cule éclairé }\end{array}$ & 41 & 6 & 47 \\
\hline $\begin{array}{l}8 \text { et } 9 \text { septembre } \\
1972\end{array}$ & $\underset{\text { bivouac }}{\operatorname{Reg} » \text { proche du }}$ & $\begin{array}{l}\text { Pièges lumineux } \\
(10 \text { pièges adhésifs : } \\
3 \mathrm{~h})\end{array}$ & 71 & 14 & 85 \\
\hline 9 septembre 1972 & Palmeraie & $\begin{array}{l}\text { Pièges lumineux } \\
(5 \text { pièges adhésifs ; } \\
3 \text { h) }\end{array}$ & 2 & 8 & 10 \\
\hline
\end{tabular}

En 1972, lors des captures réalisées le long du transect Meknès - Merzouga - Ouarzazate, $S$. antennata a surtout été observé en compagnie de (tableau IV): $P$. papatasi (34\%), S. fallax $(25 \%)$ et $P$. alexandri $(20 \%)$. Une telle association, caractéristique du bioclimat saharien, confirme, si besoin était, les affinités tropico-sahariennes du complexe $S$. antennata.

TABleau IV. - Espèces associées à Sergentomyia antennata (Newst.) le long du «transect» MeknèsMerzouga-Ouarzazate

\begin{tabular}{|c|c|c|c|c|c|}
\hline Espèces & $q$ & 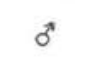 & Total & $\begin{array}{l}\text { Densités } \\
\left(\mathrm{Phl} / \mathrm{m}^{2}\right)\end{array}$ & $\%$ \\
\hline Phlebotomus papatasi ........ & 410 & 1667 & 2077 & 15,34 & 34,01 \\
\hline Sergentomyia fallax ........... & 718 & 836 & 1554 & 11,47 & 25,45 \\
\hline Phlebotomus alexandri ....... & 136 & 1112 & 1248 & 9,21 & 20,44 \\
\hline Sergentomyia antennata ...... & 305 & 313 & 618 & 4,56 & 10,12 \\
\hline Sergentomyia minuta s. sp. .... & 89 & 166 & 255 & 1,88 & 4,18 \\
\hline Phlebotomus longicuspis ...... & 15 & 147 & 162 & 1,20 & 2,65 \\
\hline Phlebotomus sergenti ......... & 25 & 61 & 86 & 0,63 & 1,41 \\
\hline Phlebotomus chabaudi ....... & 8 & 31 & 39 & 0,20 & 0,64 \\
\hline Sergentomyia christophersi .. & 14 & 22 & 36 & 0,27 & 0,59 \\
\hline Sergentomyia africana...$\ldots \ldots$ & . & 16 & 16 & 0,12 & 0,26 \\
\hline Sergentomyia dreyfussi ....... & 4 & 4 & 8 & 0,06 & 0,13 \\
\hline Phlebotomus bergeroti ....... & 3 & 3 & 6 & 0,04 & 0,10 \\
\hline Phlebotomus mariae...$\ldots \ldots$ & . & 1 & 1 & 0,01 & 0,02 \\
\hline Sergentomyia clydei .......... & . & 1 & 1 & 0,01 & 0,02 \\
\hline
\end{tabular}




\begin{tabular}{|c|c|c|c|c|c|c|c|c|c|c|c|c|c|c|c|}
\hline 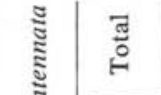 & $\dot{H}$ & F & ה & 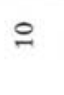 & $\stackrel{\varrho}{=}$ & $=$ & F & $=$ & त & సి & $=$ & $\nabla$ & $N$ & 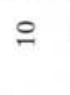 & - \\
\hline \multirow{2}{*}{ 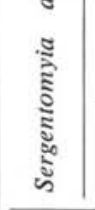 } & $N$ & $a$ & $N$ & 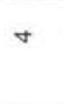 & $\approx$ & $r$ & $\tilde{\lambda}$ & $a$ & 0 & $n$ & $\cdot$ & $m$ & $\cdot$ & $\nabla$ & . \\
\hline & N & $\stackrel{\infty}{\infty}$ & . & 0 & 寸 & 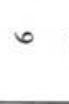 & สิ & $\infty$ & $\stackrel{\infty}{\sim}$ & ন & $=$ & - & $N$ & 0 & - \\
\hline 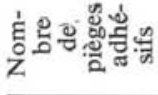 & 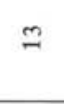 & สิ & 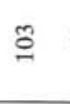 & R & $r$ & $\stackrel{9}{=}$ & $\simeq$ & $\underline{\hat{s}}$ & \pm & 3 & $n$ & ন & జి & $r$ & జి \\
\hline 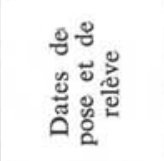 & \multicolumn{5}{|c|}{ 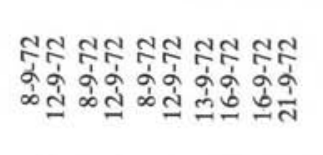 } & \multicolumn{10}{|c|}{ 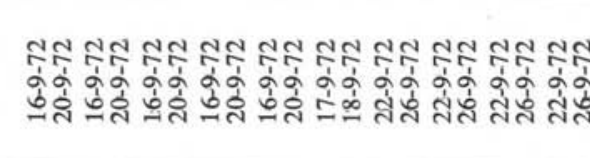 } \\
\hline 㟧 & 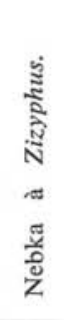 & 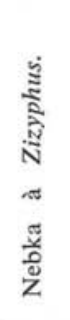 & 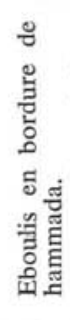 & 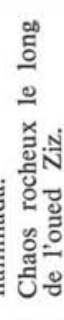 & 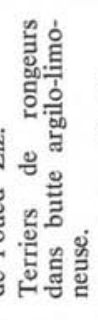 & 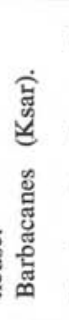 & 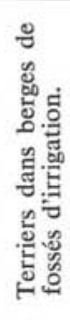 & 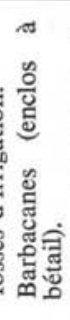 & 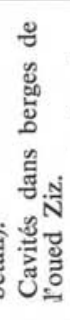 & 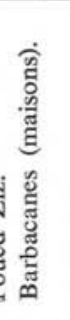 & 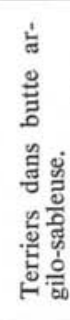 & 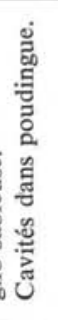 & 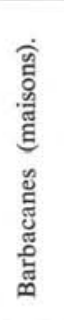 & 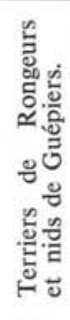 & 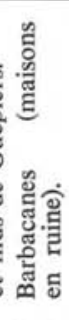 \\
\hline 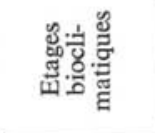 & कै & 崩 & $\ddot{\omega}$ & 㞫 & $\vec{\omega}$ & $\vec{\omega}$ & $\vec{n}$ & $\vec{n}$ & $\vec{w}$ & $\vec{\omega}$ & $\vec{\infty}$ & 㞫 & 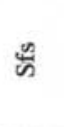 & 峁 & 峁 \\
\hline 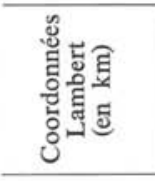 & 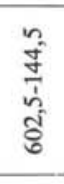 & 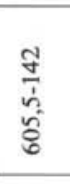 & $\stackrel{\sim}{\frac{\sim}{ \pm}}$ & $\begin{array}{l}\frac{1}{0} \\
\frac{n}{n} \\
\frac{1}{6}\end{array}$ & 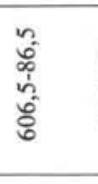 & $\begin{array}{l}\infty \\
\infty \\
1 \\
n \\
n \\
0\end{array}$ & 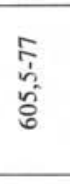 & $\begin{array}{l}n \\
\frac{n}{5} \\
\vdots \\
0\end{array}$ & $\frac{\pi}{5}$ & $\begin{array}{l}n \\
0 \\
0 \\
n \\
0 \\
0 \\
0\end{array}$ & $\begin{array}{l}\bar{n} \\
\text { ปे } \\
\tilde{6}\end{array}$ & 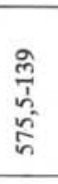 & $\frac{\infty}{\frac{n}{d}}$ & 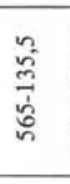 & 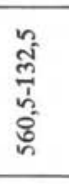 \\
\hline $\begin{array}{l}\text { 롤 } \\
\text { 娄령 }\end{array}$ & § & \&̊요 & ถి & స్ని & $\stackrel{\&}{\sim}$ & $\stackrel{\triangleright}{\curvearrowright}$ & $\stackrel{2}{\lessgtr}$ & $\stackrel{\&}{\circ}$ & $\stackrel{?}{\lessgtr}$ & $\stackrel{尺}{\vDash}$ & గ్ర & $\stackrel{\&}{\circ}$ & $\stackrel{8}{8}$ & $\stackrel{ }{=}$ & $\stackrel{\text { ㅇ }}{=}$ \\
\hline 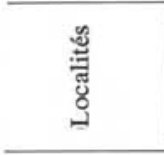 & 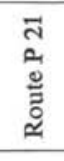 & $\begin{array}{l}\bar{N} \\
\stackrel{a}{a} \\
\tilde{o} \\
\tilde{a}\end{array}$ & 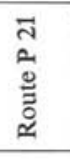 & 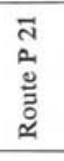 & 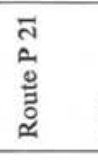 & సี & 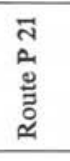 & 范 & 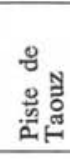 & 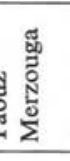 & 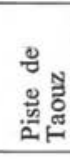 & 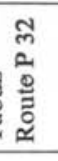 & 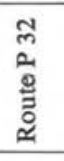 & 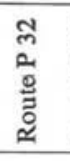 & 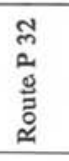 \\
\hline \multirow{2}{*}{ 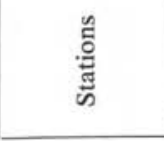 } & ร & $\stackrel{b}{*}$ & in & $\curvearrowleft$ & in & $\stackrel{\infty}{n}$ & nิ & 8 & $\overline{6}$ & ్ㅣ & 8 & $\ddot{0}$ & ฉิ & $\stackrel{2}{\circ}$ & $F$ \\
\hline & $\sum_{i}$ & $\sum_{k}$ & $\sum_{H}$ & $\sum_{H}$ & $\underset{H}{\sum}$ & $\sum_{H}$ & $\sum_{H}$ & $\sum_{H}$ & $\sum_{i}$ & $\sum_{H}^{\Sigma}$ & $\sum_{i}$ & $\sum_{i}$ & $\sum_{H}$ & $\sum_{H}$ & $\sum_{H}$ \\
\hline
\end{tabular}




\begin{tabular}{|c|c|c|c|c|c|c|c|c|c|c|c|c|c|c|c|c|}
\hline$\frac{\text { ปี }}{2}$ & $\cong$ & 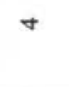 & $m$ & $\approx$ & $\vartheta$ & F & - & $N$ & - & - & $N$ & + & - & $N$ & $N$ & m \\
\hline$\stackrel{\Xi}{\Xi}$ & 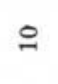 & - & - & $\stackrel{\Xi}{\mathrm{O}}$ & స్ల & $=$ & . & $N$ & - & - & . & . & . & $\cdot$ & - & . \\
\hline ڤัँ & N & m & $N$ & $\vec{n}$ & $=$ & $\bar{m}$ & - & . & . & . & N & + & - & $N$ & - & $m$ \\
\hline 之े & $a$ & 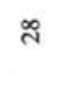 & ส & 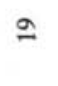 & กี & ్ㅡㅁ & J & $\vec{\pi}$ & ㅇ & 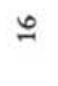 & $\cong$ & $\infty$ & ร & 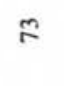 & $\stackrel{ }{\varrho}$ & $\stackrel{\infty}{\curvearrowright}$ \\
\hline 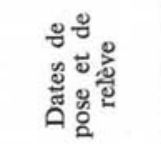 & \multicolumn{16}{|c|}{ 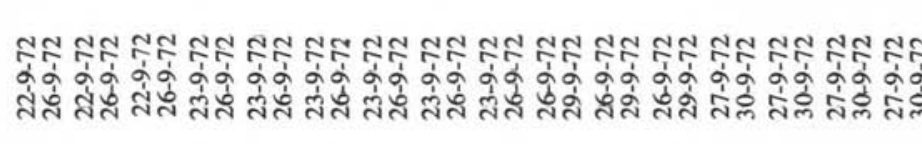 } \\
\hline ڤัٌ & 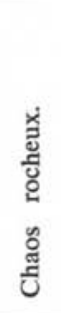 & \multicolumn{2}{|c|}{ 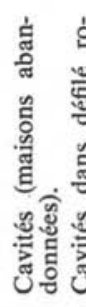 } & 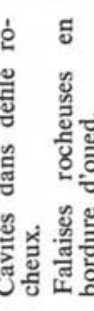 & 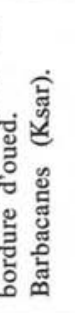 & \multicolumn{2}{|c|}{ 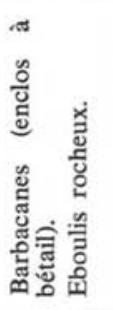 } & 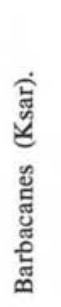 & \multicolumn{2}{|c|}{ 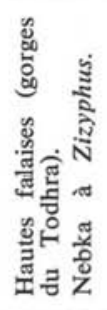 } & 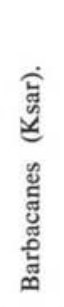 & \multicolumn{2}{|c|}{ 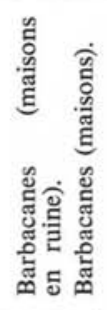 } & \multicolumn{2}{|c|}{ 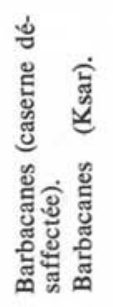 } & 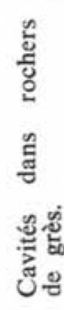 \\
\hline 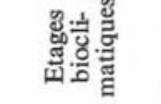 & $\frac{n}{\omega}$ & $\ddot{n}$ & $\ddot{n}$ & $\frac{n}{\omega}$ & $\ddot{\omega}$ & $\ddot{n}$ & $\ddot{n}$ & $\ddot{n}$ & Ј & $\ddot{n}$ & $\frac{n}{\omega}$ & $\ddot{n}$ & $\ddot{\omega}$ & $\ddot{n}$ & $\ddot{n}$ & मै \\
\hline 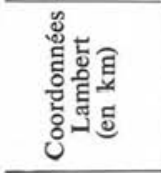 & $\frac{n}{m_{b}^{0}}$ & 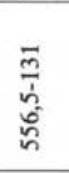 & $\frac{n}{\stackrel{n}{ \pm}}$ & $\frac{n}{\frac{1}{n}}$ & 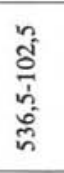 & $\frac{\delta}{\delta}$ & 号 & $\begin{array}{l}\infty \\
\infty \\
\infty \\
\sigma\end{array}$ & 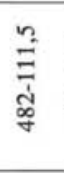 & \begin{tabular}{l}
$n$ \\
$\infty$ \\
$\infty$ \\
$\stackrel{+}{+}$ \\
\multirow{+}{*}{}
\end{tabular} & $\frac{n}{\frac{a}{\sigma}}$ & $\frac{n}{\frac{9}{9}}$ & 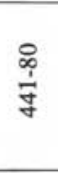 & $\frac{\tilde{v}}{\frac{\tilde{v}}{\tilde{y}}}$ & $\begin{array}{l}n \\
\frac{n}{n} \\
\frac{1}{4} \\
\text { aे }\end{array}$ & $\begin{array}{l}\text { fo } \\
\frac{n}{n} \\
\frac{n}{n}\end{array}$ \\
\hline 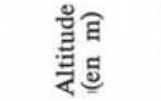 & $\stackrel{n}{\leftrightarrows}$ & $\stackrel{\text { g }}{=}$ & $\stackrel{8}{=}$ & ஃু & \& & \& & $\stackrel{8}{0}$ & 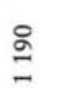 & $\stackrel{\wp}{\longleftarrow}$ & ల్ల & $\underset{\text { สิ }}{-}$ & 导 & 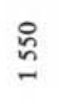 & $\stackrel{\wp}{\longleftarrow}$ & $\stackrel{\text { : }}{\text { I }}$ & $\stackrel{ }{3}$ \\
\hline 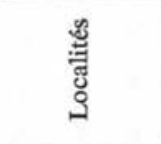 & 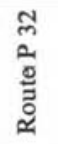 & 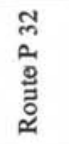 & 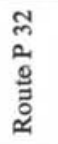 & 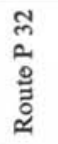 & 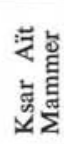 & 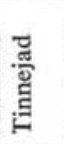 & 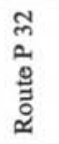 & 若息 & 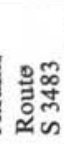 & लू & 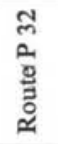 & 茝 & 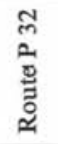 & 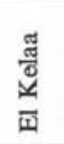 & 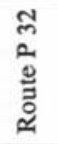 & $\begin{array}{l}\text { N } \\
\stackrel{2}{g} \\
\stackrel{g}{0} \\
\stackrel{0}{\approx}\end{array}$ \\
\hline \multirow{2}{*}{ 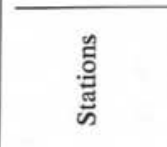 } & $\mathbb{N}$ & $\approx$ & $\approx$ & $\stackrel{\infty}{\sim}$ & 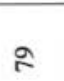 & \& & $\bar{\infty}$ & డు & $\infty$ & $\begin{array}{l}\frac{n}{3} \\
\infty \\
\infty\end{array}$ & ळ & $\bar{a}$ & 5 & 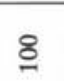 & $\cong$ & 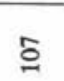 \\
\hline & $\sum_{H}$ & $\sum_{H}$ & $\sum_{L}$ & $\sum_{H}$ & $\sum_{K}$ & $\sum_{H}$ & $\sum_{L}$ & $\sum_{H}$ & $\sum_{H}$ & $\sum_{H}$ & $\sum_{H}$ & $\sum_{H}$ & $\sum_{H}$ & $\sum_{H}$ & $\sum_{H}$ & $\sum_{H}$ \\
\hline
\end{tabular}




\begin{tabular}{|c|c|c|c|c|c|c|c|c|c|c|c|c|c|c|}
\hline$\stackrel{\Xi}{\Xi}$ & J & m & 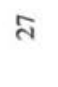 & $\stackrel{\infty}{n}$ & 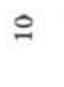 & $n$ & $N$ & $\tilde{q}-n$ & ${ }^{0}=$ & - & $\bullet$ & $a \stackrel{\infty}{=}$ & - & + \\
\hline : & 6 & . & $m$ & $=$ & $\infty$ & $\forall$ & $\cdot$ & $m \cdot \cdot$ & in & $\cdot$ & $m$ & Na & - & $\cdot$ \\
\hline 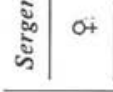 & $\stackrel{\infty}{m}$ & $m$ & A & $F$ & $N$ & 一 & $N$ & gे $-\mathrm{N}$ & 00 & - & $m$ & -2 & - & $\nabla$ \\
\hline$\stackrel{\varrho}{\tilde{g}}$ & 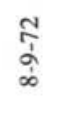 & 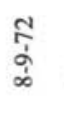 & $\frac{\hat{a}}{a}$ & $\frac{2}{\alpha}$ & $\frac{2}{\alpha}$ & $\frac{1}{2}$ & 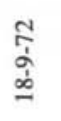 & 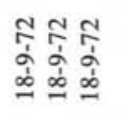 & 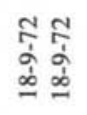 & 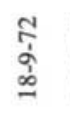 & $\frac{1}{\mathfrak{2}}$ & 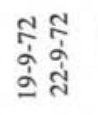 & ते & $\frac{9}{\frac{9}{2}}$ \\
\hline 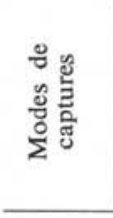 & $\begin{array}{l}\text { ๊ } \\
\text { हूँ } \\
\text { हू } \\
\text { हैं }\end{array}$ & 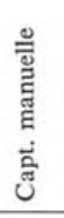 & 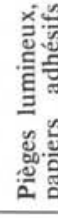 & 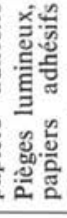 & 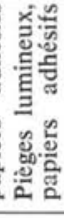 & 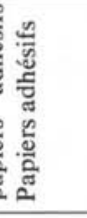 & 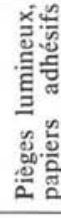 & 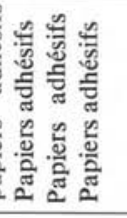 & 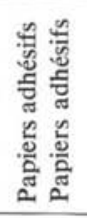 & 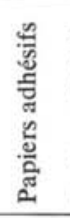 & 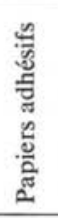 & 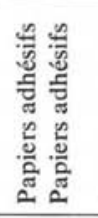 & 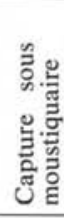 & 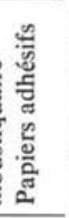 \\
\hline $\begin{array}{l}00 \\
\frac{0}{0} \\
\stackrel{0}{0}\end{array}$ & 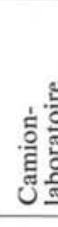 & 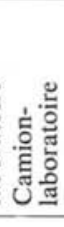 & बू & ๕̈ & 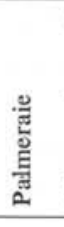 & 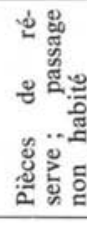 & 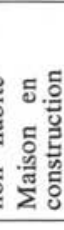 & 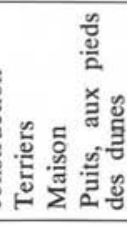 & 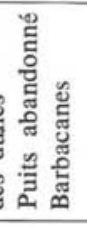 & 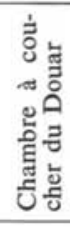 & 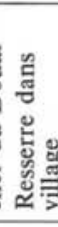 & 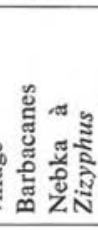 & 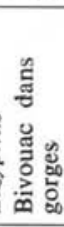 & 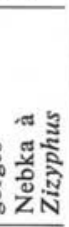 \\
\hline 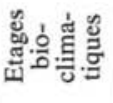 & $\ddot{n}$ & 峁 & $\stackrel{\infty}{n}$ & $\ddot{\omega}$ & ڤึ & $\ddot{n}$ & $\ddot{n}$ & $\vec{n} \vec{n} \ddot{\omega}$ & $\vec{n} \vec{n}$ & $\vec{n}$ & $\vec{n}$ & $\vec{\omega} \ddot{\omega}$ & ङ & $\ddot{n}$ \\
\hline 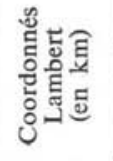 & $\frac{0}{3}$ & $\stackrel{0}{\frac{1}{6}}$ & $\stackrel{0}{\frac{1}{6}}$ & $\stackrel{0}{\frac{1}{6}}$ & $\frac{0}{\frac{1}{6}}$ & $\frac{0}{\frac{1}{6}}$ & $\begin{array}{l}0 \\
0 \\
0 \\
n \\
0 \\
0 \\
6\end{array}$ & 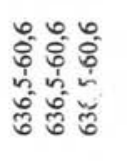 & 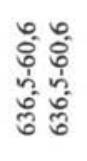 & $\begin{array}{l}0 \\
80 \\
0 \\
\hat{0} \\
0 \\
0\end{array}$ & $\begin{array}{l}0 \\
0 \\
0 \\
1 \\
0 \\
0 \\
0 \\
0\end{array}$ & 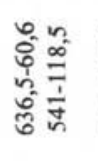 & 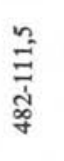 & $\begin{array}{l}\text { के } \\
\text { के } \\
\text { ఫे }\end{array}$ \\
\hline 当迳运 & ஜి & ஜ̊ & ஜ & ஜ & ஜ & ํㅗ & 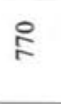 & 양 & $\stackrel{2}{\vDash}$ & $\stackrel{2}{\vDash}$ & 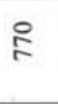 & 옹음 & ๖̊ & ๓̊ \\
\hline 氖 & 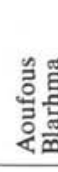 & 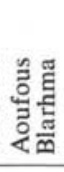 & 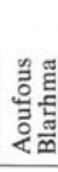 & 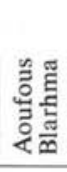 & 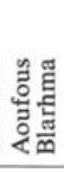 & 总 & 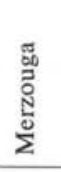 & 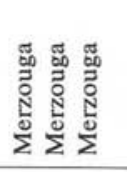 & 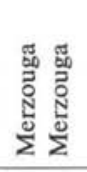 & 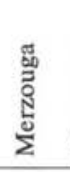 & 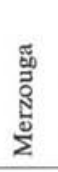 & 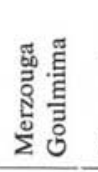 & 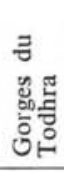 & 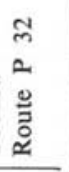 \\
\hline \multirow{2}{*}{ בั๊ } & 요 & ल & N & $\approx$ & $\hat{n}$ & 8 & $\stackrel{\infty}{=}$ & తేさ さ & 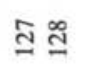 & పి & $\bar{m}$ & $\Xi \tilde{\Xi}$ & 娄 & 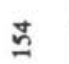 \\
\hline & $\sum_{a}$ & $\sum_{2}$ & $\sum_{a}$ & $\sum_{a}$ & $\sum_{a}$ & $\sum_{a}$ & $\sum_{a}$ & $\sum_{a} \sum_{a} \sum_{a}$ & $\sum_{a} \sum_{a}$ & $\sum_{a}$ & $\sum_{a}$ & $\sum_{a} \sum_{a}$ & $\sum_{a}$ & $\sum_{a}$ \\
\hline
\end{tabular}




\section{Bibliographie}

Abonnenc (E.), 1958. - Morphologie de Phlebotomus antennatus Newstead, 1912 et de Phlebotomus dubius Parrot, Mornet et Cadenat, 1945 d'après des individus d'élevage. Arch. Inst. Pasteur Algér., 36, 314-326.

Durand-Delacre (R.) et Memin (Y.), 1953. - Les Phlébotomes des terriers de rongeurs sauvages au Sahara. Arch. Inst. Pasteur Algér., 31, 320-333.

KIRK (R.) et Lewis (D. J,), 1949. - Taxonomy of the ethiopian sandflies (Phlebotomus). IV. P. bedfordi Newstead and P. antennatus Newstead. Ann. trop. Med. Parasit., $43,333-336$.

Larousse (F.), 1921. - Etude systématique et médicale des Phlébotomes. Thèse de médecine, Paris, 103 p.

LEWIS (D. J.) et KIRK (R.), 1951. - The sandflies (Phlebotominae) of the Anglo-Egyptian Sudan. Bull. ent. Res. 41, 563-575.

Newstead (R.), 1912. - Notes on Phlebotomus with descriptions of new species. Part. I. Bull. entom. Res., 3, 361-367.

- 1920. - On the genus Phlebotomus. Part. IV. Bull. entom. Res., 11, 305-311.

- et Sinton (J. A.), 1921. - On a collection of Papataci flies (Phlebotomus) from India. Ann. trop. Med. Parasit., 15, 103-106.

Parrot (L.), 1930. - Sur une collection de Phlébotomes du Congo belge. Revue Zool. Bot. afr., 19, 181-191.

—, 1942. - Notes sur les Phlébotomes. XXXIX. A propos de deux Prophlebotomus d'Algérie: Phlebotomus minutus var. signatipennis et Phlebotomus fallax. Arch. Inst. Pasteur Algér., 20, 322-335.

—, 1948. - Notes sur les Phlébotomes. LIX. Phlehotomus du Soudan anglo-égyptien. Arch. Inst. Pasteur Algér. 26, 259-276.

—, 1951. - Notes sur les Phlébotomes. LXII. Phlebotomus dubius, Phlebotomus antennatus var. cinctus et leur distribution géographique. Arch. Inst. Pasteur Algér., 29, 129. 133.

- et Durand-Delacre (R.), 1947. - Notes sur les Phlébotomes LVI. Phlébotomes de Béni-Ounif-de-Figuig (Sahara oranais). Arch. Inst. Pasteur Algér., 25, 77-86.

— et Martin (L.), 1944. - Notes sur les Phlébotomes. XLIV. Phlébotomes de Djibouti. Arch. Inst. Pasteur Algér., 22, 55-58.

—, Mornet (P.) et Cadenat (J.), 1945. - Notes sur les Phlébotomes. XLVIII. Phlébotomes de l'Afrique occidentale francaise. I. Sénégal, Soudan, Niger. Arch. Inst. Pasteur Algér., 23, 232-244.

- Mornet (P.) et Cadenat (J.), 1945. - Notes sur les Phlébotomes. L. Phlébotomes de l'Afrique occidentale Française. 2. Guinée, Côte-d'Ivoire, Dahomey. Arch. Inst. Pasteur Algér., 23, 281-289.

-, et Picheyre (R.), 1941. - Notes sur les Phlébotomes. XXXVIII. Phlébotomes du Hoggar. Arch. Inst. Pasteur Algér., 19, 441-442. 
Rioux (J.-A.), Golvan (Y.-J.), Houin (R.), Croset (H.), Juminer (B.), Bain (O.) et Tour (S.), 1967. - Ecologie des leishmanioses. 1. - Les Phlébotomes. Echantillonnage. Ethologie. Ann. Paras. Hum. Comp. 42, 561-603.

Sinton (J. A.), 1928. - The synonymy of the Asiatic species of Phlebotomus. Indian J. med. Res., 16, 297-324.

SCHWEtZ (J.), 1940. - Note supplémentaire sur la répartition des Phlébotomes au Congo belge. Revue Zool. Bot. afr., 33, 241-244.

THEOdor (O.), 1931. - On african sandflies. Bull. entom. Res., 22, 469-478.

—, 1933. - Some african sandflies. Bull. entom. Res., 24, 537-547. 